\title{
Using MCMC Probit Model to Value Coastal Beach Quality Improvement
}

\author{
Zuozhi Li, Erda Wang, Jingqin Su, Yang Yu
}

School of Management, Dalian University of Technology, Dalian, China.

Email: LZZ200603@mail.dlut.edu.cn, edwang@dlut.edu.cn,jingqin@dlut.edu.cn,yuyang770727@hotmail.com

Received revised October $18^{\text {th }}, 2010$; revised November $25^{\text {th }}, 2010$; accepted December $29^{\text {th }}, 2010$.

\begin{abstract}
Dichotomous choice elicitation technique of contingent valuation method is broadly used in the research fields of environmental resource and recreational activity management. The binary choice type of questions are generally analyzed by using Logit or Probit probability distribution models in which a common analysis procedure is to apply MLE for estimating variable parameters before calculating the respondents' willingness to pay. In this paper, a MCMC Gibbs sampling Probit model is adopted to maintain the three advantages it has in dealing with heteroscedasticity, high dimension numerical integral and sample size restriction problems. The results revealed that the MCMC model and MLE Probit model are strikingly consistent, which suggests that the former is much simple and reliable estimation method. At the same time, the empirically based existence value estimation of coastal beach quality improvement in Dalian, China is $R M B ¥ 168$ per person.
\end{abstract}

Keywords: Value of Coastal Beach Quality, Markov Chain Monte Carlo (MCMC), Probit Model

\section{Introduction}

Contingent valuation technique is commonly used in environmental resource management fields, and monetized benefit measurement for improvements of environmental goods. Abundant research in beach nourishment has been conducted by applied economists [1-4]. In this paper, we are primarily concerned over the subject of coastal beach quality improvement with a particular emphasis on the relationship between tourism beach nourishment and tourists' socioeconomic characteristics and the nature of their tourism activities as well. Beach conditions such as slope, width, mud, debris, congestion etc. are easily observable and perceptively recognized by the tourists through photos presented to them. Tourists' responses to the improved situation of coastal tourism resource quality is depended on a sequence of bidding value data to let respondent decide "Yes" or "No" regarding acceptance or rejection in an empirical survey. The type of binary dependent variable model in the empirical study can be estimated utilizing Logit or Probit probability distribution which is firstly explored by Bishop and Heberlein (1979) with dichotomous choice (DC) elicitation model of CVM [5], which is called single-bounded DC.

Funding through the National Natural Science Foundation of China (NNSFC) with Approval No 70871014
The theoretical base of this method used in consumer welfare measurement is analyzed by Hanemann (1984) using Random Utility Maximization (RUM) [6]. With the support of the US Department of Commerce's National Oceanic and Atmospheric Administration (NOAA) [7], RUM based utility difference DC model has been widely accepted and broadly extended. Traditionally, parameterized method of OLS or MLE is used, but it requires satisfying with homoscedastic assumption in order to be able to carry out a regression procedure in OLS, as well as a high dimension numerical integral which sometimes causes difficulty in solving consumer surplus based on MLE. Therefore, in this paper, we try to examine the performance of the non-parametric kernel heteroscedastic MCMC method by comparing its results against those generated by MLE in order to reveal the robustness of MCMC Gibbs sampling method.

\section{Probit Regression Models}

\subsection{Heteroscedastic Regression Model}

Bayesian treatment of the independent student $t$ linear model, in which, variances of random error are embodied in heteroscedastic characteristics broken through the traditional model to ensure a better statistical fitness (Geweke, 1993) [8]. The useful model is 


$$
\begin{aligned}
& \quad y_{i}=\boldsymbol{x}_{i}^{\prime} \boldsymbol{\beta}+\varepsilon_{i}, \varepsilon_{i} \square N\left(0, \sigma^{2} \omega_{i}\right)(i=1, \cdots, n) \\
& \text { Or } \\
& \boldsymbol{y}=\boldsymbol{X} \boldsymbol{\beta}+\boldsymbol{\varepsilon}, \operatorname{var}(\boldsymbol{\varepsilon})=\sigma^{2} \boldsymbol{\Omega}, \boldsymbol{\Omega} \equiv \operatorname{diag}\left(\omega_{1}, \cdots, \omega_{n}\right) \\
& \boldsymbol{y}_{n \times 1}=\left(y_{1}, \cdots, y_{n}\right)^{\prime} \\
& \boldsymbol{X}_{n \times k}=\left[\boldsymbol{x}_{1}, \cdots, \boldsymbol{x}_{n}\right]^{\prime} \\
& \boldsymbol{\varepsilon}=\left(\varepsilon_{1}, \cdots, \varepsilon_{n}\right)^{\prime} \\
& \boldsymbol{\omega}=\left(\omega_{1}, \cdots, \omega_{n}\right)^{\prime}
\end{aligned}
$$

And the likelihood function is

$$
\begin{aligned}
& L(\boldsymbol{\beta}, \boldsymbol{\sigma}, \boldsymbol{\omega} ; \boldsymbol{y}, \boldsymbol{X})=(2 \pi)^{-n / 2} \cdot \sigma^{-n} \\
& \quad \prod_{i=1}^{n}\left\{\omega_{i}^{-1 / 2} \cdot \exp \left[-\sum_{i=1}^{n}\left(y_{i}-\boldsymbol{x}_{i}^{\prime} \beta\right)^{2} /\left(2 \sigma^{2} \omega_{i}\right)\right]\right\}
\end{aligned}
$$

According to (1), there exist three hyper-parameters, and then the prior density is

$$
\pi(\boldsymbol{\beta}, \sigma, \omega)=\pi_{1}(\boldsymbol{\beta}) \cdot \pi_{2}(\sigma) \cdot \pi_{3}(\omega)
$$

Bayesian Markov chain Monte Carlo (MCMC) method is a comprehensive approach to solve high dimension numerical integral, because the analytical solution of exact posterior moments cannot be done, in the paper, the Gibbs sampler is used to draw a sequence of data. To use $\mathrm{MCMC}$, the key point is to find the conditional posterior distributions. Hyper-parameter vector $\omega$ is not finally focused on, and it can be transformed into a single hyper-parameter $v$ to assigns an independent $\chi^{2}(v) / v$ prior distribution to $\omega_{\mathrm{i}}$ terms. Furthermore, the $\mathrm{jth}$ drawing data set is based on $\boldsymbol{\theta}^{(j)}=\left\{\boldsymbol{\beta}^{(j)^{\prime}}, \sigma^{(j)}, \boldsymbol{\omega}^{(j)^{\prime}}, v^{(j)}\right\}$. In his study, the conditional posterior distributions are

$$
\begin{gathered}
\boldsymbol{\beta} \mid(\sigma, \boldsymbol{\omega}) \square N(\Delta, \Lambda) \\
\Delta=\left[\boldsymbol{X}^{\prime} \boldsymbol{\Omega}^{-1} \boldsymbol{X}+\sigma^{2} \boldsymbol{G}^{\prime} \boldsymbol{T}^{-1} \boldsymbol{G}\right]^{-1}\left[\boldsymbol{X}^{\prime} \boldsymbol{\Omega}^{-1} \boldsymbol{y}+\sigma^{2} \boldsymbol{G}^{\prime} \boldsymbol{T}^{-1} \boldsymbol{g}\right] \\
\Lambda=\sigma^{2}\left[\boldsymbol{X}^{\prime} \boldsymbol{\Omega}^{-1} \boldsymbol{X}+\sigma^{2} \boldsymbol{G}^{\prime} \boldsymbol{T}^{-1} \boldsymbol{G}\right]^{-1} \\
\quad\left[\sum_{i=1}^{n}\left(u_{i}^{2} / \omega_{i}\right) / \sigma^{2}\right] \mid(\beta, \omega) \square \chi^{2}(n) \\
{\left[\left(\sigma^{-2} u_{i}^{2}+v\right) / \omega_{i}\right] \mid(\beta, \sigma) \square \chi^{2}(v+1)}
\end{gathered}
$$

\subsection{Probit Parameter Method}

Probit model is one of binary choice probability distribution models, which is usually used to solve dichotomous choice models of contingent valuation method (CVM) in valuing environmental resource or leisure activities. For binary choice model, given sampling from $i=1$ to $\mathrm{n}$, the explicit dependent variable is evaluated " 0 " or " 1 " as $z_{i}$ in (6), which is representing the relationship between implicit dependent variable of continuous distribution as $y_{i}$ in (6) and explanation variables. Its basic format is as (6).

$$
z_{i}=\left\{\begin{array}{l}
0 \text { if } y_{i} \leq 0 \\
1 \text { if } y_{i}>0
\end{array}\right.
$$

In benefit measurement for improvements of tourism goods, tourists are asked information of willingness to pay (WTP) for environment or policy change. The WTP is a bidding value (explanation variable $\boldsymbol{B I D}$ in this paper), furthermore, consumer surplus or environmental improvement value is measured through mean or median of WTP probability distribution after estimating parameters mentioned $\boldsymbol{\beta}$ above. Based on the utility difference model by Hanemann (1984), the implicit dependent variable $y_{i}$ explained with a vector of independent variables $\mathrm{x}_{i}$ can be considered as the utility difference, and his mentioned utility difference model is like (1) [6]. According to his RUM assumption, given bidding value B, there is the probability expression as (7).

$$
P(\text { "Yes" })=P(B \leq W T P)=P\left(\varepsilon_{i} \leq y_{i}\right)=F\left(y_{i}\right)
$$

Traditional probability distribution of random error is paid attention to Logit or Probit model $[5,6]$. In Probit model, error accords with $\mu=0$ and $\sigma^{2}=1$ standard Normal distribution, whose cumulative density function (CDF) is as (8).

$$
P\left(\text { "Yes") }=P\left(z_{i}=1 \mid W T P\right)=\Phi\left(y_{i}\right)\right.
$$

Integrated (7) with (8), WTP's probability density function (PDF) and CDF could be written as Equation (9) and (10).

$$
\begin{aligned}
f(x)=\left(-\beta_{x}\right) & (1 / \sqrt{2 \pi}) \exp \left[-(\boldsymbol{X} \boldsymbol{\beta})^{\prime}(\boldsymbol{X} \boldsymbol{\beta}) / 2\right] \\
P\left(z_{i}=1, i\right. & =1, \cdots, n \mid x)=\Phi(x) \\
& =1-\int_{-\infty}^{\mathbf{X} \boldsymbol{\beta}}(1 / \sqrt{2 \pi}) \exp \left(-t^{2} / 2\right) d t
\end{aligned}
$$

Through MLE function (11), coefficient parameters $\boldsymbol{\beta}$ can be estimated.

$$
\ln L=\sum_{i=0}^{n}\left\{z_{i} \ln [\Phi(x)]+\left(1-z_{i}\right) \ln [1-\Phi(x)]\right\}
$$

\subsection{MCMC Probit Regression Model}

In Probit model, $\varepsilon_{i}$ is distributed as standard normal distribution, and so in Gibbs sampling, a sequence of processes of normal distribution sampling for $y i$ are appeared. Due to (6), the left or right zero-truncated normal-distributed sampling is used to get posterior values. In each sampling, based upon (6) and (1) as step (b) be- 
low, all of $\mathrm{n}$ original samples $\left(y_{i}, i=1\right.$ to $\left.\mathrm{n}\right)$ are revalued for estimating the hyper-parameters.

The Gibbs sampling MCMC steps can be organized as follows.

1) The initial value $\left[\boldsymbol{\beta}^{(0)},\left(\sigma^{2} \Omega\right)^{(0)}\right]$ is conveniently assigned using OLS results with $\boldsymbol{\beta}^{(0)}=\left(\boldsymbol{X}^{\prime} \boldsymbol{X}\right)^{-1} \boldsymbol{X}^{\prime} \boldsymbol{y}$, $\left[\left(\sigma^{2} \boldsymbol{\Omega}\right)^{(0)}\right]=(\boldsymbol{y}-\boldsymbol{X} \boldsymbol{\beta})^{\prime}(\boldsymbol{y}-\boldsymbol{X} \boldsymbol{\beta}) /(n-k)$, as well as $\boldsymbol{\omega}^{(0)}=(1,1, \cdots, 1)_{1 \times n}^{\prime}$ and $v=200$ is a fixed value for simplifying calculation.

2) Given $\boldsymbol{\theta}^{(j)}=\left\{\boldsymbol{\beta}^{(j)^{\prime}}, \sigma^{(j)}, \boldsymbol{\omega}^{(j)^{\prime}}, v^{(j)}\right\}$ to sample in step j. Referred to $z_{i}, i=1, \cdots, n$ in (6) to sample $\boldsymbol{y}^{(j)}$ from normal distribution for left zero-truncation if $z_{i}=1$ and right zero-truncation if $z_{i}=0$.

3) Draw $\boldsymbol{\beta}^{(j+1)}$ conditional on $\boldsymbol{\sigma}^{(j)}$ and $\boldsymbol{\omega}^{(j)}$ using $\boldsymbol{\beta}^{(j)}$ and (3), which means calculating the mean and variance of $\boldsymbol{\beta}^{(j+1)}$ depended on $\boldsymbol{\theta}^{(j)}$ in last loop.

4) Draw $\sigma^{(j+1)}$ conditional on $\beta^{(j+1)}$ and $\boldsymbol{\omega}^{(j)}$ using (4), which means deciding $\sigma^{(j+1)}$ in (4) after computing $\varepsilon^{(j+1)}=\boldsymbol{y}^{(j+1)}-\left(\boldsymbol{x}^{\prime}\right)^{(\boldsymbol{j}+1)} \boldsymbol{\omega}^{(j+1)}$, randomly drawing $\chi^{2}(n)$ and using $\omega^{(j)}$.

5) Draw $\omega^{(j+1)}$ conditional on $\beta^{(j+1)}$ and $\boldsymbol{\sigma}^{(j+1)}$ using (5), which means deciding $\omega^{(j+1)}$ in (5) after computing $\boldsymbol{\varepsilon}^{(\boldsymbol{j}+1)}=\boldsymbol{y}^{(\boldsymbol{j}+1)}-\left(\boldsymbol{x}^{\prime}\right)^{(\boldsymbol{j}+1)} \boldsymbol{\omega}^{(j+1)}$, randomly drawing $\chi^{2}(v+1)$ and using $\boldsymbol{\sigma}^{(j+1)}$.

6) Express a sampling route from step (2-5), repeat the route 3000 times, and obtain Markov chain $\{s 1001$, $s 1002, \cdots, s 3000\}$ after deleting the first 1000 set of data.

In this process, a Bayesian regression approach is presented for the chief purpose of getting hyper parameters $\boldsymbol{\beta}$. According to the steps above, the Mean $\left[\boldsymbol{\beta}^{(1001)}, \boldsymbol{\beta}^{(1002)}\right.$, $\left.\cdots, \boldsymbol{\beta}^{(3000)}\right]$, a point estimator of $\boldsymbol{\beta}$, can be easily achieved. Hereto the estimated $\boldsymbol{\beta}$ of MCMC approach together with $\boldsymbol{\beta}$ of MLE point estimator are obtained in use to value WTP of coastal beachquality improvement below.

\section{WTP Estimators}

Transform Equation (1) to (12) after retaining variable $\boldsymbol{B I D}$ and calculating the remainder to get estimated constant $\alpha$ replaced explained variables with respective means.

$$
\hat{y}=\hat{\alpha}+\hat{\beta}_{x} x
$$

Using the symmetrical property of the PDF curve for a standard normal distribution, we can set up $F(y)=0.5$ in (7), from which the WTP median of equation (12) can be computed based on (13). Using Equations of (9) and (12), WTP mean can be obtained as through Equation (14).

$$
\begin{gathered}
\operatorname{Median}(W T P)=-\hat{\alpha} / \hat{\beta}_{x} \\
\operatorname{Median}(W T P)=\int_{-\infty}^{+\infty} X \cdot\left(-\hat{\beta}_{x}\right) \cdot(1 / \sqrt{2 \pi}) \\
\exp \left[-\left(\hat{\alpha}+\hat{\beta}_{x} x\right)^{2} / 2\right] d x=-\hat{\alpha} / \hat{\beta}_{x} x
\end{gathered}
$$

\section{Statistical Analyses}

\subsection{Variables and Empirical Data}

Tourism site survey was conducted from September 25 to October 10 in 2009 lasting for 15 days. Of which one week (Oct.1 - 7) is the Chinese national holiday that is so-called "golden week" period. The selected survey sites included four primary tourism sites in Dalian of northeastern China, including Tiger Beach Park, Fujiazhuang Bathing Beach, Xinghai Square Beach, and Xinghai Park. A pilot survey was conducted for pretest purpose before a formal interview survey was implemented. The survey sampled 1276 individual tourists, of which 1 206 observations are valid responses after eliminating those incomplete survey response questionnaire. Thus, the effective survey response rate was $94.5 \%$. The statistical descriptive information is listed in Table 1.

Variable $\boldsymbol{Y A N}$ is binary choice dependent variable which is specified as ' 1 ' given a "Yes" response, and ' 0 ' for "No" response. $\boldsymbol{B I D}$ is the priority variable which represents WTP of individual interviewee and it is labeled as 7-levels of payment including $¥ 5, Ұ 10, Ұ 20$, $¥ 50, ¥ 100, ¥ 200$ and $¥ 500$. To fulfill with DC type of questionnaire design requirements, the number of tourists surveyed correspondent to each level of WTP is approximately equivalent. Variable $\boldsymbol{A} \boldsymbol{G E}$ is specified 1-5 categories, in which ' 1 ' = below 15 , ' 2 ' = above 15 and below 25, ' 3 ' = above 25 and below 40, '4' = above 40 and below 60, and ' 5 ' = above 60. Variable $\boldsymbol{E D U}$ (Education) is characterized 6 categories: ' 1 ' = elementary school graduate and below, ' 2 ' $=$ high or vocational school graduate, ' 3 ' = junior college graduate, ' 4 ' = college or university graduate, ' 5 ' = master, and ' 6 ' = doctor. Variable INC (Income) is specified as 1 to 10 categories: (US\$ $1=$ RMB¥6.83) ' 1 ' = less than or equal to 500 , ' 2 ' = 500-999, ' 3 ' = 1,000-1,999, '4' = 2,000-2,999, '5'= $3,000-3,999$, ' 6 ' = 4,000-5,999, '7' = 6,000-7,999, ' 8 ' = $8,000-10,000, ' 9$ ' $=10,000-20,000$, and ' 10 ' $=$ greater than 20,000. Variable $\boldsymbol{R} \boldsymbol{K} \boldsymbol{M}$ represents a respondent's round trip travel distance $(\mathrm{km})$. Variable $\boldsymbol{S} \boldsymbol{W}, \boldsymbol{V G}, \boldsymbol{F G}$, $\boldsymbol{Y T}$, and $\boldsymbol{S P}$ are recreational activity variables which respectively stands for swimming, sea-sighting and strolling, fishing, yachting, and play game on the sand. Each of them is also characterized as ' 0 ' or ' 1 ' dummy variable. 
Table 1. The variables and descriptive statistics of survey data.

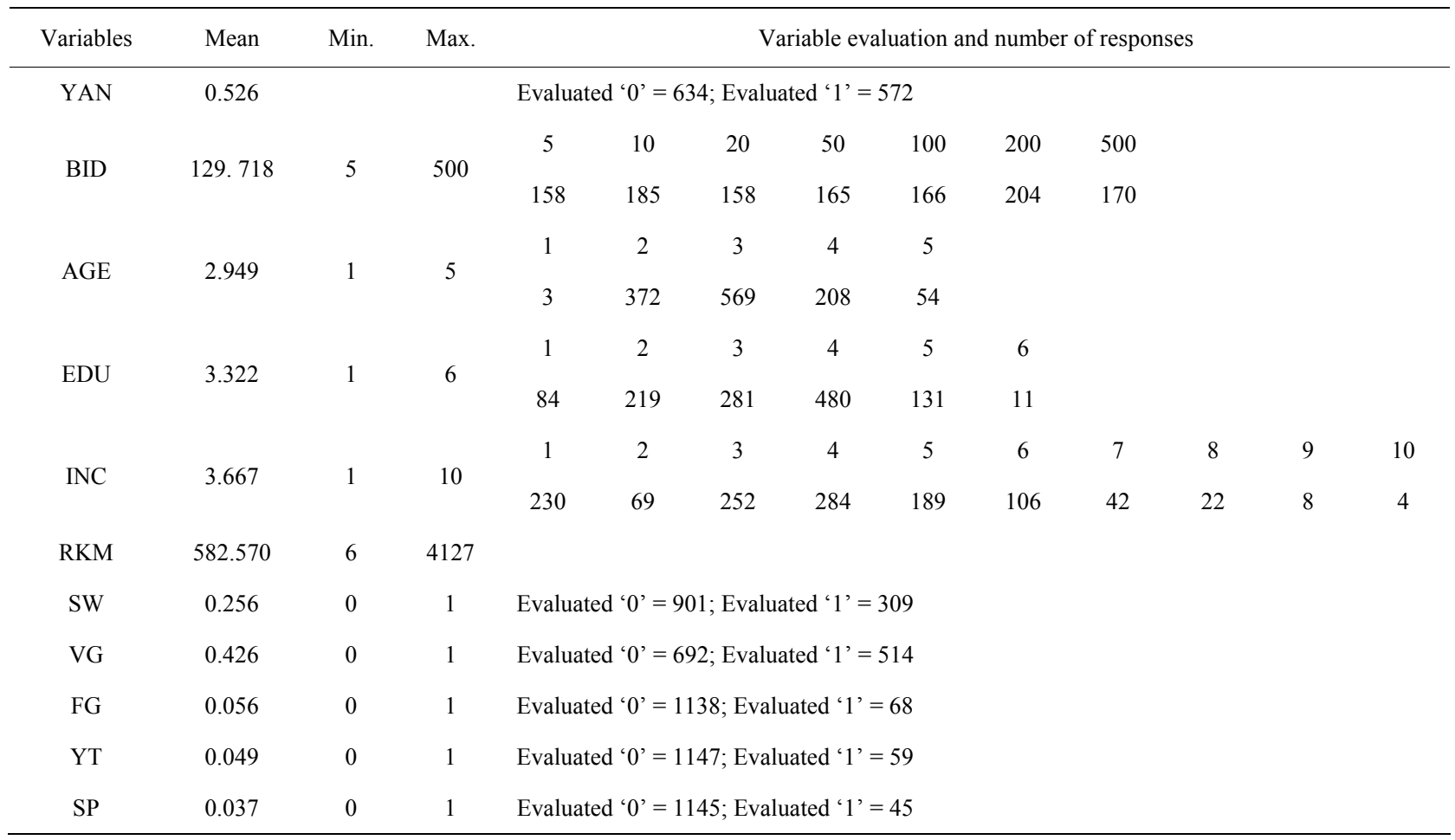

\subsection{Results and Discussion}

Based on Equations of (12), (13) and (14), the median WTP and mean WTP can be computed through using explanation variables show in Table 2, respectively, where $\operatorname{Mean}\left(W T P_{M L E}\right)=\operatorname{Median}\left(W T P_{M L E}\right)=166.99$ and $\operatorname{Mean}\left(W T P_{M C M C}\right)=\operatorname{Median}\left(W T P_{M C M C}\right)=168.20$.

In Table 2, statistical tested were conducted to evaluate the regression model performance. It turns out that there are three asymptotically equivalent statistical tests including the likelihood ratio statistic, the Wald statistic, and the Lagrange multiplier statistic, which can measure goodness-of-fit and joint significance of all coefficients except the constant, but McFadden pseudo $\mathrm{R}^{2}$ and the likelihood ratio test are commonly used. In these two models, the test results are almost identical with each other. McFadden pseudo $\mathrm{R}^{2}$ and the likelihood ratio can be used to evaluate a model's goodness-of-fits, and they all reach excellent level of significance in both models. T-test indicates that all explanatory variables reach 0.10 or better statistical significance except for variables of $\boldsymbol{S} \boldsymbol{W}$ and $\boldsymbol{S P}$. These highly consistent results suggest that the MCMC Probit model is effective method for DCCVM type of analysis.

In estimating for both MLE parameters and hyper-parameters of MCMC, two different models were used and both got consistent $\mathrm{t}$-statistics as $\mathrm{p}$-value indicated, which suggest that all those main estimators are acceptable for the WTP estimations. Thus, in case a researcher is mainly interested in the study procedure to deal with complicated problems, a non-parameter estimation program should be recommended.

Connotation reflected through coefficients of explanatory variables transmits the information that $\boldsymbol{B I D}, \boldsymbol{A G E}$, and activities variables are negatively correlated with latent utility difference. The negative signs of coefficients have different meaning for WTP measurement because $\boldsymbol{B I D}$ 's is a denominator and the other explanatory variables are embedded in the numerator of WTP calculation. Coefficient modulus of $\boldsymbol{B I D}$ expresses an inverse ratio relationship with WTP, while the other explanatory variables are truly negative correlations with WTP.

\section{Conclusions}

In this paper, a MCMC Gibbs sampling Probit model is used to evaluate the coastal beach quality improvement. From an empirical study perspective, the procedure can be used to estimate economic value for multiple study fields including but not limited to healthy, risk, transportation, resources and environment and ecology compensation, etc. In comparison with traditional MLE Probit model, MCMC Probit model achieves very consistent results, which suggest that the WTP estimation using both methods is more robust. 
Table 2. Probit model estimation and comparison.

\begin{tabular}{ccccccc}
\hline & & MLE Probit Model & & & \multicolumn{2}{c}{ MCMC Probit Model } \\
\cline { 2 - 7 } Variable & Coefficient & t-statistic & $\mathrm{p}$ & Coefficient & t-statistic & p \\
\hline Const & 0.670 & 3.010 & 0.003 & 0.678 & 3.034 & 0.002 \\
BID & -0.003 & -12.271 & 0.000 & -0.003 & -12.842 & 0.000 \\
AGE & -0.141 & -2.645 & 0.008 & -0.143 & -2.647 & 0.004 \\
EDU & 0.066 & 1.807 & 0.071 & 0.066 & 0.038 \\
INC & 0.050 & 2.166 & 0.031 & 0.051 & 2.157 & 0.008 \\
RKM & 0.000 & -1.576 & 0.115 & 0.000 & -1.585 & 0.062 \\
SW & -0.097 & -0.785 & 0.433 & -0.101 & -0.824 & 0.198 \\
VG & -0.204 & -1.801 & 0.072 & -0.205 & -1.784 & 0.036 \\
FG & -0.365 & -1.959 & 0.050 & -0.374 & -1.979 & 0.027 \\
YT & -0.338 & -1.714 & 0.087 & -0.339 & -1.717 & 0.046 \\
SP & -0.144 & -0.733 & 0.464 & -0.147 & -0.726 & 0.238 \\
McFadden Pseudo R2 & & & & & & 0.1176
\end{tabular}

As well as, a MCMC Gibbs sampling model holds better qualities because of the three advantages in dealing with heteroscedasticity, high dimension numerical integral and sample size restriction problems. Parametric model based on OLS or MLE test procedures is widely applied for estimation of consumer surplus although the existence of heteroscedasticity does not fit in OLS and a high dimension numerical integral is also difficult to be solved using traditional parametric method. The nonparametric model using MCMC has been gradually recognized as a handy method for estimating regression models. In the process, applying the MCMC Gibbs sampling to get hyper-parameters estimators is well received $[9,10]$. However, it is worth of noting the importance that it is necessary to get the conditional posterior distributions.

The authors used the utility difference model to estimate environmental resources via comparing and estimating a non-parametric regression model with 2000 enlarged samplers. Analyzed the regression coefficients, the finding is that education and income represent similar elasticity implication, which is the opposite of age and activities. The result indicates that the existence value of the coastal beach environmental quality improvement in Dalian, China is RMB ¥168 per person, and this result is so consistent with the one obtained from using a traditional method.

\section{REFERENCES}

[1] V. K. Smith, X. Zhang and R. B. Palmquist, "Marine Debris, Beach Quality, and Non-Market Values," Environmental and Resource Economics, Vol. 10, No. 3, 1997, pp. 223-247. doi:10.1023/A:1026465413899

[2] J. C. Whitehead, C. F. Dumas, J. Herstine, J. Hill and B. Buerger, "Valuing Beach Access and Width with Revealed and Stated Preference Data," Marine Resource Economics, Vol. 23, No. 2, 2008, pp. 119-135.

[3] C. E. Landry, A. G. Keeler and W. Kriesel, "An Economic Evaluation of Beach Erosion Management Alternatives," Marine Resource Economics, Vol. 18, No. 2, 2003, pp. 105-127.

[4] C. Oh, A. W. Dixon, J. W. Mjelde and J. Draper, "Valuing Visitors' Economic Benefits of Public Beach Access Points," Ocean and Coastal Management, Vol. 51, No. 12,2008 , pp. 847-853. doi:10.1016/j.ocecoaman.2008.09.003

[5] R. C. Bishop and T. A. Heberlein, "Measuring Values of Extra-market Goods: Are Indirect Measures Biased," American Journal of Agricultural Economics, Vol. 61, No. 5, 1979, pp. 926-930. doi:10.1016/j.ocecoaman.2008.09.003

[6] W. M. Hanemann, "Welfare Evaluations in Contingent Valuation Experiments with Discrete Responses," American Journal of Agricultural Economics, Vol. 66, No. 3, 1984, pp. 332-341.

[7] R. Arrow, R. Solow, P. Portney, E. Leamer, R. Radner 
and H. Schuman, "Report of the NOAA Panel on Contingent Valuation," 1993.

http://www.darrp.noaa.gov/library/pdf/cvblue.pdf

[8] J. Geweke, "Bayesian Treatment of the Independent Student t Linear Model," Journal of Applied Econometrics, Vol. 8, No. 1, 1993, pp.19-40.

doi:10.1002/jae.3950080504

[9] S. Chib and E. Greenberg, "Markov Chain Monte Carlo
Simulation Methods in Econometrics," Econometric Theory, Vol. 12, No. 3, 1996, pp. 409-431. doi:10.1017/S0266466600006794

[10] R. E. Kass, B. P. Carlin, A. Gelman, and R. M. Neal, "Markov Chain Monte Carlo in Practice: A Roundtable Discussion," The American Statistician, Vol. 52, No. 2, 1998, pp. 93-100. doi:10.2307/2685466 